\section{Utilização de genéricos em área de atuação da equipe de Saúde da Família em município do sul do Brasil}

\section{Utilization of generics in an area covered by a family health unit in a southern Brazilian city}

Milene Zanoni da Silva Vosgerau'

Regina Kazue Tanno de Souza"

Darli Antonio Soares" (in memoriam)

'Universidade Federal do Paraná

"Universidade Estadual de Londrina

\section{Resumo}

Esta pesquisa transversal objetivou estimar a prevalência do uso de genéricos na população total e entre os usuários de medicamentos; verificar as classes medicamentosas mais consumidas entre os genéricos; avaliar o nível de conhecimento dos genéricos pela população; e identificar os fatores associados ao uso de genéricos entre adultos de 20 a 59 anos. A amostra foi composta por 374 indivíduos da área de abrangência de uma Unidade Saúde da Família de Ponta Grossa, PR. A coleta de dados foi feita por entrevistas domiciliares. Foram consideradas três categorias de variáveis: sociodemográficas, condição de saúde e uso de serviços de saúde. Para análise estatística foi utilizado o teste de qui-quadrado. A prevalência de consumo de genéricos foi de 9,9\%. Dos entrevistados, 96,5\% afirmaram conhecer os genéricos; $64,3 \%$ acreditam que estes têm a mesma qualidade e $88,9 \%$ relataram que são mais baratos. Os grupos mais utilizados foram os do sistema nervoso e do aparelho cardiovascular. A quase totalidade $(96,2 \%)$ dos genéricos foi adquirida em farmácias comerciais. Após análise univariada, as variáveis estatisticamente significativas foram classificação econômica, situação de trabalho, plano de saúde, presença de doenças crônicas, consulta nos últimos 3 meses e internação nos últimos 12 meses. A baixa prevalência de utilização aponta para a necessidade do fortalecimento de políticas de disponibilização de genéricos na rede pública, em especial na Estratégia Saúde da Família, como principal forma de acesso a esses medicamentos à população como a estudada.

Palavras-chave: Medicamentos genéricos. Utilização de medicamentos. Farmacoepidemiologia. Estudos transversais. Adulto. Programa Saúde da Família. 


\section{Abstract}

The objective of the study was to estimate the prevalence of generics and their associated factors in a population of adults aged 20 to 59 years. The sample consisted of 374 individuals from the catchment area of a Family Health Unit in Ponta Grossa, PR, Brazil. Data were collected in home interviews. Study variables were divided into 3 groups: sociodemographic, health status and the use of health services. The chi-square test was used for statistical analysis. The prevalence of generics was $9.9 \%$. Of those interviewed, 96.5\% claimed to know about generic drugs, $64.3 \%$ believed they had the same quality as brand-name drugs and $88.9 \%$ reported they were cheaper. The most commonly-used groups of drugs were those for the nervous system and those for the cardiovascular system. After univariate analysis, the following factors were found to be statistically significant: economic status, employment status, health insurance, presence of chronic disease, medical consultation in the previous three months and hospitalization within the previous 12 months. The low prevalence of the use of generic drugs emphasizes the fact that stronger policies are needed to make generics available to the public, especially through the Family Health Strategy, since it is the studied population's principal form of access to these medications.

Keywords: Generic drugs. Drug utilization. Pharmacoepidemiology. Cross-Sectional Studies. Adult. Family Health Program.

\section{Introdução}

O acesso a produtos farmacêuticos seguros e eficazes é fundamental na promoção do uso racional de medicamentos. Em decorrência do elevado custo dos medicamentos e deficiência na regulação do mercado farmacêutico, estima-se que 2 bilhões de pessoas, um terço da população mundial, não têm acesso regular a medicamentos essenciais, comprometendo muitas vezes a terapêutica instituída ${ }^{1}$.

Dentre as estratégias para a promoção do acesso a fármacos, encontra-se a política de medicamentos genéricos, que foi implantada no Brasil em 1999, com a promulgação da Lei $9787^{2}$. Esta política introduziu novos conceitos e definiu o medicamento genérico como "produto farmacêutico intercambiável", produzido após a expiração da proteção patentária ou outros direitos de exclusividade, e que deve ser equivalente ao produto inovador ${ }^{2}$. Além dos medicamentos genéricos, o mercado brasileiro atualmente apresenta medicamentos de referência e similares. Os medicamentos de referência, também conhecidos como inovadores ou de marca, cuja eficácia, segurança e qualidade foram comprovadas cientificamente junto ao órgão federal competente no momento do registro ${ }^{3}$, são os produtos que se encontram há bastante tempo no mercado $\mathrm{e}$ têm uma marca comercial conhecida. Os similares são medicamentos que possuem o mesmo fármaco, a mesma concentração, forma farmacêutica, via de administração, posologia e indicação terapêutica do medicamento de referência, contudo não apresentam o teste de bioequivalência comprovado.

Após a promulgação da lei, foram publicados diversos decretos, portarias e resoluções com o objetivo de facilitar o registro, regulamentar a produção, normatizar os testes de qualidade e de bioequivalência, além de estimular o comércio de genéricos no país. A consolidação da política de medicamentos genéricos se baseou em algumas ações principais, tais como: extinção do similar com nome de princípio ativo, criação 
da embalagem diferenciada para genéricos, campanhas para a população e ações de promoção dirigidas à classe médica ${ }^{4,5}$.

Ao revisar a literatura sobre os genéricos no Brasil, de 1999 até hoje, encontram-se principalmente estudos de biodisponibilidade e bioequivalência de medicamentos, diferenças de preços entre as especialidades farmacêuticas, além de estudos qualitativos das representações sociais de profissionais da saúde e usuários acerca dos genéricos. Contudo, existem poucas investigações em relação aos fatores que influenciam a utilização dos medicamentos genéricos.

Dentre os estudos epidemiológicos encontrados, os principais aspectos que têm sido analisados são a prevalência de utilização de genéricos ${ }^{6}$, fatores sociodemográficos associados ao seu consumo ${ }^{6}$ e conhecimento da população acerca dos genéricos ${ }^{6-8}$. Investigações sobre a prevalência de uso de genéricos e fatores associados são necessários, principalmente em áreas de atuação do Programa Saúde da Família (PSF), estratégia estruturante dos sistemas municipais de saúde, reordenador do modelo de atenção no SUS, que atualmente cobre $49,9 \%$ da população brasileira ${ }^{9}$.

A identificação da situação de uso e dos conhecimentos sobre os genéricos pela população assistida pela estratégia Saúde da Família pode contribuir no direcionamento de ações voltadas a promover maior acesso a esses medicamentos e, consequentemente, à terapia farmacológica. Assim, os objetivos desta pesquisa foram: estimar a prevalência do uso de genéricos na população total e entre os usuários de medicamentos; verificar as classes medicamentosas mais consumidas entre os genéricos, avaliar o nível de conhecimento dos genéricos pela população e identificar os fatores associados ao uso de genéricos entre adultos acompanhados por uma Unidade Saúde da Família.

\section{Material e métodos}

Estudo transversal desenvolvido em área de abrangência de uma Unidade Saúde da Família de Ponta Grossa, Paraná. A população alvo de estudo foi constituída de moradores com idades entre 20 e 59 anos completos. O tamanho da amostra, para uma margem de erro de $5 \%$ e um nível de confiança de $95 \%$, foi de 361 indivíduos. A este número se adicionou $20 \%$ para eventuais perdas, totalizando, assim, 434 pessoas a serem entrevistadas.

Os serviços de saúde de Ponta Grossa, na atenção primária, são formados por 46 unidades básicas de saúde. Destas, 40 estão na região urbana, contando com 28 equipes de Saúde da Família. A Estratégia Saúde da Família está implantada desde 2001 e inicialmente contava com um farmacêutico como membro integrante da equipe mínima. À época da realização do estudo, somente a Unidade de Saúde da Família (USF) em estudo manteve a presença do farmacêutico.

Os dados para a seleção da amostra foram coletados junto à base de dados do Sistema de Informação da Atenção Básica (SIAB), por meio de amostragem sistemática. A unidade de observação foi o indivíduo. Para o cálculo do intervalo amostral, dividiu-se o número total de indivíduos dentro da faixa etária estipulada pelo tamanho da amostra. O intervalo foi igual a 14 .

O pré-teste deu-se a partir de moradores da área não incluídos na amostra da pesquisa. O instrumento utilizado para a coleta de dados foi um formulário estruturado, composto por 58 questões referentes ao aspecto sociodemográfico, à condição de saúde, ao uso de serviços de saúde e, especificamente, a utilização de medicamentos genéricos. $\mathrm{O}$ conhecimento foi verificado com a pergunta: "Você conhece ou ouviu falar de medicamento genérico?".

Os participantes do estudo foram questionados acerca de todo medicamento em uso, definido previamente como aqueles utilizados pelo entrevistado nos últimos sete dias que antecederam à entrevista. Para analisar a utilização de medicamentos intercambiáveis foram considerados os entrevistados que consumiram pelo menos um produto genérico.

As entrevistas foram realizadas entre 
dezembro de 2006 e janeiro de 2007 por equipes previamente treinadas, organizadas em quatro duplas, compostas por um acadêmico do curso de farmácia e outro de enfermagem. Dúvidas, inconsistências e itens não preenchidos foram devidamente discutidos com o(a) entrevistador(a) e averiguados pela pesquisadora envolvida na coleta de dados. Consideraram-se como perdas as pessoas não encontradas em até oito tentativas de contato, em horários e dias da semana diferentes.

A variável dependente da pesquisa foi o uso de medicamentos genéricos nos últimos sete dias que antecederam a entrevista. As variáveis independentes foram: sexo, idade, escolaridade, situação de trabalho, nível econômico - conforme poder de compra da família segundo a Associação Brasileira de Empresas e Pesquisa (ABEP) ${ }^{10}$-, autopercepção de saúde, presença auto-referida de enfermidades crônicas, filiação a plano de saúde, consulta com médico nos últimos três meses e internação hospitalar nos últimos doze meses.

O nível econômico foi categorizado de acordo com os critérios estabelecidos pela ABEP, que envolve posse de bens e o nível de escolaridade do chefe da família, permitindo a classificação em cinco estratos, sendo que o estrato A representa a melhor condição financeira e o estrato E a pior situação.

Para a validação do uso de medicamentos, foi utilizada a metodologia padrão instituída por Landry et al. (1988) ${ }^{11}$. Tal técnica consiste em solicitar aos entrevistados a embalagem, receita, bula e blister de produtos farmacêuticos utilizados, com o objetivo de evitar omissão, em geral por esquecimento, de medicamentos em uso e, além disso, para assegurar a veracidade das especialidades farmacêuticas informadas ${ }^{12}$. Os nomes e laboratórios fabricantes foram devidamente registrados para posterior classificação em grupos farmacológicos.

Os princípios ativos presentes em cada especialidade foram listados e organizados de acordo com a classificação ATC (Anatomical Therapeutical Chemical), elaborada pelo "Nordic Council on Medicines" e reco- mendada pela "Drug Utilization Research Group" (DURG) da OMS para os Estudos de Utilização de Medicamentos ${ }^{13}$. Os insumos com mais de um princípio ativo foram classificados na classe terapêutica do principal componente; produtos com diferentes ações farmacológicas foram enquadrados levando-se em conta sua indicação terapêutica.

As informações anotadas nos formulários foram duplamente digitadas. Para o procedimento de validação, os bancos foram comparados e, após correção, obtevese o banco de dados definitivo. O programa do Epi Info, versão 3.3.2 para Windows foi utilizado tanto na entrada como na análise dos dados. Para a análise de associação das variáveis independentes com o consumo de medicamento genérico utilizou-se o teste de qui-quadrado. Consideraram-se significativas as associações que apresentaram valores correspondentes a $\mathrm{p}<0,05$.

Esta pesquisa teve aprovação do Comitê de Ética da Universidade Estadual de Londrina (CAAE 0210.0.268.000-06). Após a apresentação dos entrevistadores, a leitura do termo de consentimento livre e esclarecido e a realização da entrevista, foi entregue um folheto que continha informações sobre o uso correto de medicamentos bem como sobre a identificação, características e qualidade dos medicamentos genéricos.

\section{Resultados}

Dos 434 indivíduos selecionados foram entrevistadas 374 pessoas $(86,2 \%)$ e registraradas 60 perdas $(13,8 \%)$. A média de idade foi de 39,5 anos (desvio padrão $=11,0$ ), sendo que $53,5 \%(n=200)$ eram do sexo feminino. Somente $2,1 \%$ eram analfabetos e 43,6\% não completaram o primeiro grau. A maioria das pessoas $(62,3 \%)$ trabalhava no mercado formal ou informal e $49,7 \%$ dos participantes foram classificados no nível econômico C da ABEP.

A prevalência de consumo de medicamentos nos últimos sete dias foi de $67,1 \%$ (IC 95\%; 62,1-71,8) $(\mathrm{n}=251)$. Ao considerar os 374 entrevistados no denominador, a 
proporção de indivíduos que utilizaram pelo menos um medicamento genérico na última semana foi de 9,9\% (IC 95\%; 7,1-13,5). Entre os 251 entrevistados que consumiram medicamentos, a prevalência de uso de genéricos foi de $14,7 \%$.

Com relação aos medicamentos utilizados pelos entrevistados, verificou-se um total de 533 especialidades farmacêuticas que puderam ser confirmadas por meio da bula, blister ou embalagem. Notou-se que $65,8 \%(\mathrm{n}=351)$ eram similares, $24,4 \%(\mathrm{n}=$ 130) eram de referência e $9,8 \%(n=52)$ eram genéricos. Com relação ao local de aquisição dos medicamentos genéricos, $96,2 \%(n=50)$ foram comprados em farmácias comerciais.

Dos 52 genéricos verificados, $48 \%$ ( $\mathrm{n}=$ 25) faziam parte da Relação Nacional de Me- dicamentos Essenciais (RENAME) ${ }^{14}$ e 51,9\% ( $\mathrm{n}=27$ ) constavam na Relação Municipal de Medicamentos Essenciais (REMUME) ${ }^{15}$. Nesta pesquisa, de todos os medicamentos obtidos na farmácia da Unidade Básica de Saúde ( $n=146$ ) em estudo, $92,5 \%$ eram similares.

Na Tabela 1 está distribuída a frequência dos medicamentos genéricos e nãogenéricos consumidos nos últimos sete dias segundo a classificação ATC. Do total de genéricos verificados $(n=52)$, a maior proporção de uso foi observada no grupo anátomo-terapêutico do sistema nervoso (32,7\%), do aparelho cardiovascular (25\%), dos antiinfecciosos gerais para uso sistêmico $(13,5 \%)$ e do aparelho digestivo e metabolismo (13,5\%). As classes farmacológicas

Tabela 1 - Distribuição dos medicamentos genéricos e não-genéricos utilizados nos últimos sete dias segundo classe terapêutica em residentes na área de uma Unidade Saúde da Família, Ponta Grossa, PR, dezembro de 2006 e janeiro de 2007.

Table 1 - Distribution of generic and non-generic drugs taken during the past seven days according to their therapeutic class in residents in a Family Health Unit area, in Ponta Grossa, PR, from December/2006 to January/2007.

\begin{tabular}{lcccc}
\hline Classe terapêutica & \multicolumn{2}{c}{ Genéricos } & \multicolumn{2}{c}{ Não-genéricos } \\
\cline { 2 - 5 } N Sistema nervoso & $\mathrm{n}$ & $\%$ & $\mathrm{n}$ & $\%$ \\
N02 Analgésicos & $\mathbf{1 7}$ & $\mathbf{3 2 , 7}$ & $\mathbf{1 2 0}$ & $\mathbf{2 4 , 9}$ \\
N03 Antiepilépticos & 11 & 21,2 & 54 & 11,2 \\
N06 Psicoanalépticos & - & - & 17 & 3,5 \\
N05 Psicolépticos & 2 & 3,8 & 22 & 4,6 \\
Outros & 3 & 5,8 & 17 & 3,5 \\
C Aparelho cardiovascular & 1 & 1,9 & 10 & 2,1 \\
C03Diuréticos & $\mathbf{1 3}$ & $\mathbf{2 5 , 0}$ & $\mathbf{1 1 0}$ & $\mathbf{2 2 , 9}$ \\
C07 Betabloquadores & 1 & 1,9 & 35 & 7,3 \\
C08 Bloqueadores dos canais de cálcio & 1 & 1,9 & 19 & 4,0 \\
C09 Agentes que atuam sobre o sistema renina-angiotensina & 4 & 7,7 & 5 & 1,0 \\
C10 Hipolipemiantes & 4 & 7,7 & 34 & 7,1 \\
Outros & 2 & 3,8 & 9 & 1,9 \\
J Antiinfecciosos gerais para uso sistêmico & 1 & 1,9 & 8 & 1,6 \\
J01 Antibacterianos para uso sistêmico & $\mathbf{7}$ & $\mathbf{1 3 , 5}$ & $\mathbf{1 3}$ & $\mathbf{2 , 8}$ \\
J05 Antivirais para uso sistêmico & 7 & 13,5 & 7 & 1,5 \\
A Aparelho digestivo e metabolismo & - & - & 6 & 1,3 \\
A10 Medicamentos usados no diabetes & 7 & 13,5 & 53 & 11,0 \\
A02 Antiácidos, medicamentos para úlcera & 6 & 11,5 & 23 & 4,8 \\
A03 Agentes antiespasmódicos, anticolinérgicos e propulsivos & 1 & - & 20 & 4,2 \\
M Sistema músculo-esquelético & $\mathbf{2}$ & $\mathbf{3}, 9$ & 10 & 2,0 \\
M01 Anti-inflamatórios e anti-reumáticos & 2 & 3,8 & 42 & 8,7 \\
Outros & 6 & 11,5 & 143 & 29,7 \\
\hline Total & 52 & 100,0 & 481 & 100,0 \\
\hline
\end{tabular}


mais consumidas foram os analgésicos (N02), os antibacterianos para uso sistêmico (J01), bloqueadores de canal de cálcio (C08), agentes que atuam sobre o sistema renina-angiotensina (C09) e medicamentos utilizados no diabetes (A10). Tanto entre os medicamentos genéricos, quanto entre os não genéricos, os medicamentos do grupo do sistema nervoso e do aparelho cardiovascular foram os mais usados.

Na Tabela 2 verifica-se a distribuição dos entrevistados sobre os conhecimentos e características dos medicamentos genéricos. Observa-se que $96,5 \%$ das pessoas afirmaram conhecer estes medicamentos; $64,3 \%$ acreditam que estes produtos têm a mesma qualidade quando comparados aos de referência; 88,9\% relataram que são mais baratos com relação aos inovadores; $\mathrm{e}$ $60,7 \%$ têm preferência pelo medicamento genérico.
A associação entre a utilização de genéricos e as variáveis demográficas, socioeconômicas, condição de saúde e uso dos serviços de saúde pode ser observada na Tabela 3. Entre os indivíduos que consumiram medicamentos, verificou-se maior prevalência de utilização de genéricos entre os indivíduos classificados nos estratos A $\mathrm{e}$ $B$ da ABEP, que não estavam trabalhando, filiados à plano de saúde, portadores de doenças crônicas, que consultaram nos últimos três meses e foram internados nos últimos 12 meses, comparativamente às categorias alternativas.

\section{Discussão}

No presente estudo, a prevalência de uso de genéricos $(9,9 \%)$ foi superior à taxa encontrada em Pelotas ( $3,6 \%)$, que utilizou um período recordatório de 15 dias $^{6}$. No en-

Tabela 2 - Distribuição dos indivíduos residentes na área de abrangência de uma Unidade Saúde da Família segundo o conhecimento e identificação das características dos medicamentos genéricos, Ponta Grossa, PR, dezembro de 2006 a janeiro de 2007.

Table 2 - Distribution of residents in the Family Health Unit area according to the awareness and identification of generics in Ponta Grossa, PR, from December/2006 to January/2007.

\begin{tabular}{lcc}
\hline & $\mathrm{n}$ & $\%$ \\
\hline Conhecimento sobre genéricos & 361 & 96,5 \\
Sim & 13 & 3,5 \\
Não & & \\
Qualidade dos genéricos & 7 & 1,9 \\
Melhor qualidade & 232 & 64,3 \\
Mesma qualidade & 40 & 11,1 \\
Menor qualidade & 82 & 22,7 \\
Não sabe & & \\
Preço dos genéricos & 321 & 88,9 \\
Mais baratos & 9 & 2,5 \\
Mesmo preço & 8 & 2,2 \\
Mais caros & 23 & 6,4 \\
Não sabe & & 60,7 \\
Preferência por medicamento & 219 & 1,9 \\
Genérico & 7 & 13,9 \\
Similar & 50 & 23,5 \\
Referência & 85 & \\
Tanto faz & &
\end{tabular}


Tabela 3 - Distribuição dos entrevistados que consumiram medicamentos segundo a utilização de genéricos conforme variáveis demográficas, socioeconômicas, condição de saúde e uso dos serviços de saúde. Ponta Grossa, PR, dezembro de 2006 a janeiro de 2007.

Table 3 -Distribution of interviewees who use medicine, according to the use of generics by demographic, social-economic variables, health conditions and utilization of health care services in Ponta Grossa, PR, from December/2006 to January/2007.

\begin{tabular}{|c|c|c|c|c|c|}
\hline \multirow[t]{2}{*}{ Variáveis } & \multicolumn{2}{|c|}{ Uso de genéricos } & \multicolumn{2}{|c|}{ Uso de não-genéricos } & \multirow[t]{2}{*}{$\mathrm{p}$} \\
\hline & $\mathbf{n}$ & $\%$ & $\mathbf{n}$ & $\%$ & \\
\hline Sexo & & & & & 0,8984 \\
\hline Masculino & 15 & 15,6 & 81 & 84,4 & \\
\hline Feminino & 22 & 14,2 & 133 & 85,8 & \\
\hline Idade (anos) & & & & & 0,3701 \\
\hline $20-39$ & 16 & 12,4 & 133 & 87,6 & \\
\hline $40-59$ & 21 & 17,2 & 101 & 82,8 & \\
\hline Grau de escolaridade & & & & & 0,3434 \\
\hline Analfabeto $/ 1^{\circ} \mathrm{grau}$ incompleto & 13 & 11,5 & 100 & 88,5 & \\
\hline $1^{\circ}$ grau completo $/ 2^{\circ}$ grau incompleto & 10 & 20,0 & 40 & 80,0 & \\
\hline $2^{\circ}$ grau completo/outros & 14 & 15,9 & 74 & 84,1 & \\
\hline ABEP & & & & & 0,0059 \\
\hline$A+B$ & 15 & 27,3 & 40 & 72,7 & \\
\hline$C+D+E$ & 22 & 11,2 & 174 & 88,8 & \\
\hline Situação de trabalho & & & & & 0,012 \\
\hline Não trabalha & 23 & 21,9 & 82 & 78,1 & \\
\hline Trabalha & 14 & 9,6 & 132 & 90,4 & \\
\hline Autopercepção de saúde & & & & & 0,0932 \\
\hline Ruim & 22 & 19,3 & 92 & 80,7 & \\
\hline Boa & 15 & 10,9 & 122 & 89,1 & \\
\hline Plano de Saúde & & & & & 0,0008 \\
\hline Sim & 20 & 27,0 & 54 & 73,0 & \\
\hline Não & 17 & 9,6 & 160 & 90,4 & \\
\hline Presença de doenças crônicas & & & & & 0,0118 \\
\hline Sim & 29 & 19,9 & 117 & 80,1 & \\
\hline Não & 8 & 7,6 & 97 & 92,4 & \\
\hline Consulta médica nos últimos 3 meses & & & & & 0,0008 \\
\hline Sim & 32 & 21,2 & 119 & 78,8 & \\
\hline Não & 5 & 5,0 & 95 & 95,0 & \\
\hline Internação hospitalar nos últimos 12 meses & & & & & 0,0000 \\
\hline Sim & 13 & 39,4 & 20 & 60,6 & \\
\hline Não & 24 & 11,0 & 194 & 89,0 & \\
\hline
\end{tabular}

tanto, cabe ressalvar que o estudo de Pelotas foi realizado em 2002, apenas 3 anos após a promulgação da Lei 9.787, que implantou a política de medicamentos genéricos no País, o que pode explicar a diferença verificada entre as prevalências. Neste período a legislação dos genéricos passava por constantes correções a fim de ampliar a utilização e conhecimento da população sobre os genéricos. Entre elas destacou-se a publicação 
das resoluções, em 2001, que colocavam o fim da comercialização dos similares sem marca e a que tornou obrigatória a inclusão do logotipo dos genéricos na embalagem externa $^{5}$.

Com relação às classes farmacológicas, o maior consumo de medicamentos genéricos de algumas classes, como os analgésicos, antibacterianos para uso sistêmico, e medicamentos utilizados no diabetes, explica-se possivelmente pelo maior número de produtos genéricos no mercado farmacêutico ${ }^{16}$. Além disso, Monteiro et al. (2005) ${ }^{17}$ identificaram que os medicamentos antibacterianos estavam entre as classes em que se verificaram maiores diferenças percentuais de preço entre o medicamento de referência e o genérico, como é o caso da amoxicilina $500 \mathrm{mg}$ cápsulas, que teve uma diferença de $56 \%$. Desta forma, a maior quantidade de especialidades farmacêuticas disponíveis nas farmácias comerciais somada aos preços mais baratos dos genéricos comparativamente aos de medicamentos de marca podem justificar os resultados encontrados na Tabela 1.

O sucesso da política de genéricos está associado à redução de preços dos medicamentos com a introdução dos genéricos. Neste sentido, autoridades sanitárias na Espanha, França, Alemanha e Estados Unidos exigiram que as indústrias produzissem genéricos a partir dos medicamentos de referência, a fim de conterem a tendência de aumento dos preços dos produtos farmacêuticos. Esta medida impactou positivamente o consumo destes produtos, já que, em 2009, a participação do mercado de genéricos nesses países, em volume, foi de $30 \%, 35 \%, 60 \%$ e $60 \%$, respectivamente ${ }^{18}$. No Brasil, o consumo de genéricos ainda é baixo comparado a outros países, apesar de ter alcançado, em poucos anos, um volume de vendas de genéricos que outros países levaram décadas para atingir ${ }^{5}$. Em 2006, os genéricos já cobriam aproximadamente $14 \%$ das vendas em unidades no mercado ${ }^{18}$.

Na população estudada, é possível que a utilização de genéricos esteja relacionada principalmente ao menor custo do medi- camento, uma vez que $88,9 \%$ dos entrevistados referiram preços mais baixos para estes produtos farmacêuticos. Entretanto, o fato de $33,8 \%$ não conhecerem ou considerarem os genéricos de menor qualidade apresenta-se como aspecto relevante. A falta de informações adequadas sobre a qualidade dos genéricos pode explicar em parte sua baixa utilização, pois nesta pesquisa um percentual menor de entrevistados $(64,3 \%)$, comparativamente ao observado pelo Ministério da Saúde (80\%), afirmou que o medicamento genérico fazia o mesmo efeito que o medicamento de referência. Sabe-se que parcela da população desconhece os testes a que os produtos genéricos são submetidos para sua aprovação pela ANVISA, o que possibilita interpretações equivocadas no sentido de se subestimar a qualidade do genérico devido ao preço mais baixo que os de marca, pois se tem fortemente arraigada a concepção popular de que "quanto mais caro melhor" ou "o que é bom custa caro"19.

Esta mentalidade é fundamentada na lógica racional, visto que nenhum outro bem de consumo no mercado mundial tem tanta diferença nos preços entre diversos produtos com as mesmas características técnicas, como acontece com os medicamentos. Tanto em países em desenvolvimento como nos desenvolvidos, os contrastes dos preços dos medicamentos chegam a ser o dobro da diferença encontrada entre alimentos e outros bens industriais ${ }^{20}$. Ressalte-se que $o$ custo dos medicamentos genéricos pode ser mais baixo pelo fato de estes "emprestarem" os resultados dos ensaios clínicos de eficácia e segurança do produto original por meio de equivalência farmacêutica (in vitro) e bioequivalência (in vivo), além de não necessitarem divulgar marcas comerciais em marketing e propaganda ${ }^{21}$.

Em Rio Branco, Faria e Tavares Neto $(2006)^{8}$, ao consideraram como "pessoas conhecedoras do medicamento genérico" aquelas que o definiram corretamente, além de identificarem sua embalagem através de um kit que continha medicamento de referência e genérico, constataram que apenas $22,1 \%$ conheciam efetivamente esses 
medicamentos. Provavelmente, os autores observaram uma taxa bem inferior a outras pesquisas pela técnica mais detalhada aplicada na avaliação do conhecimento da população, o que sugere que o conhecimento sobre genérico mencionado no presente estudo não reflete necessariamente a adequada informação sobre a sua qualidade.

A maioria $(60,7 \%)$ dos entrevistados desta pesquisa disse que os medicamentos genéricos são a primeira opção na hora da compra. No entanto, observou-se contradição entre a preferência e o uso de genéricos, que pode estar relacionada com a troca espontânea de medicamento feita pelos balconistas nas farmácias, onde na maioria das vezes os clientes não são consultados, estimulando o consumo de medicamentos similares em decorrência das bonificações dadas pelas indústrias farmacêuticas.

Este ato ilegal pode ser coibido a partir do controle efetivo dos usuários no momento da compra; entretanto, para isso são necessárias ações educativas e propagandas que sejam veiculadas pelas instituições governamentais, a fim de garantir informações objetivas e imparciais focadas nas diferenças entre genéricos e similares. Em investigação desenvolvida em Rio Branco, a própria população indicou que as campanhas educativas deveriam ocorrer nos bairros e nas Unidades de Saúde utilizando dados mais claros e de fácil compreensão ${ }^{8}$.

No presente estudo, embora a prevalência do uso de genéricos tenha sido superior entre aqueles com escolaridade mais elevada, a diferença não foi significativa. Entretanto, a prevalência de uso mais elevada entre as pessoas de melhores condições econômicas sugere que, se por um lado o preço dos genéricos, geralmente inferior aos de referência, estimula o seu uso por pessoas de melhor renda, por outro lado o maior custo, quando comparado aos similares, pode ser impedimento para a sua utilização entre os segmentos economicamente mais desfavorecidos.

Possivelmente o poder aquisitivo dos entrevistados também explique as maiores prevalências de uso de genéricos entre os filiados a planos privados de saúde, pois, conforme a Pesquisa Nacional por Amostra de Domicílios (PNAD 1998, 2003), os níveis mais elevados de renda entre adultos (2064 anos) estão positivamente associados à maior filiação a planos de saúde ${ }^{22}$.

A associação positiva entre o uso de genéricos e as hospitalizações nos últimos 12 meses, presença de doenças crônicas e consulta médica nos últimos doze meses provavelmente se relaciona ao contato mais frequente destes indivíduos com os serviços de saúde e, dessa maneira, a efetividade na comunicação sobre a qualidade dos genéricos, bem como a recomendação de seu uso por parte dos prescritores, pode ser uma hipótese a ser aventada para maior utilização verificada nestes grupos. Já os achados relacionados à situação de trabalho e genéricos carecem de outros estudos direcionados para sua melhor compreensão.

Assim, mesmo com preços reduzidos, os indivíduos de estratos econômicos menos favorecidos têm dificuldades para adquirir genéricos em farmácias comerciais em decorrência da baixa renda ${ }^{23}$; além disso, a população também não tem acesso a estes produtos pelo Sistema Único de Saúde. O fato de a quase totalidade dos genéricos $(96,2 \%)$ terem sido adquiridos em farmácias comerciais reflete situação predominante no Brasil, como também em outros países latino-americanos, nos quais se disponibilizam amplamente produtos farmacêuticos similares no setor público ${ }^{24}$. Uma experiência relevante é a do México, que exige que o setor público compre preferencialmente os medicamentos intercambiáveis para possibilitar o acesso da população a produtos mais seguros e eficazes ${ }^{25}$.

A lei das licitações e contratos públicos (8.666/93) veio para equacionar os problemas relacionados ao binômio qualidadecusto, pois permite a inserção de critérios de exigências de qualidade no momento da aquisição de produtos, inclusive os farmacêuticos. Contudo, no setor público predominam a compra de produtos restritos ao critério de menor preço, o que pode, em algumas situações, comprometer a seguran- 
ça e a efetividade dos medicamentos ditos essenciais $^{26}$.

Nesse sentido, a implantação da Lei 9.787/99 trouxe grande avanço para o sistema de saúde brasileiro, pois, além de melhores preços, a regulamentação da política de genéricos está sendo determinante para enquadrar os medicamentos similares aos mesmos padrões de qualidade exigidos aos produtos intercambiáveis. A Resolução 134/2003 da Agência Nacional da Vigilância Sanitária (ANVISA) dispõe que os medicamentos similares já comercializados devem se adequar, nos próximos 10 anos, ao objetivo de garantir segurança e qualidade por meio de ensaios de equivalência farmacêutica e biodisponibilidade relativa ${ }^{17}$.

No Brasil, são escassos os estudos publicados sobre a utilização de medicamentos genéricos e seus fatores associados. Vale ressaltar que, nas bases de dados consultadas (MEDLINE, LILACS e SCIELO), somente a investigação de Bertoldi et al. ${ }^{6}$ se assemelha à metodologia aplicada a este trabalho. Este fato indica a necessidade de realização de novos estudos para possibilitar comparações com outras populações e avançar na identificação dos aspectos que possam contribuir para o aumento do uso de genéricos no setor farmacêutico nacional, especialmente em áreas cobertas pelo Programa Saúde da Família, pois, como se evidenciou nesta pesquisa, o consumo destes produtos ainda é pequeno entre a população assistida pela estratégia.

Em relação aos aspectos metodológicos, algumas situações, conforme descrito em trabalho anterior ${ }^{27}$, foram controladas para garantir a validade interna da pesquisa. Quanto às perdas, o percentual mencionado no presente trabalho não invalida o estudo, pois no planejamento da investigação foi dado tratamento adequado e, por precaução, acrescentados $20 \%$ à amostra inicialmente calculada.

Os resultados, ao revelarem que quase a totalidade dos entrevistados expressa conhecimento sobre o genérico, sugere a difusão do produto na população. Entretanto, a baixa prevalência de uso, com diferença significativa entre os estratos de renda, e o fato da maioria desses medicamentos ter sido adquirida em farmácia comercial, aponta para a necessidade de avançar no fortalecimento do uso de genéricos, com definição de políticas, especialmente por parte dos gestores municipais, no sentido de priorizar a disponibilização de genéricos na rede pública, como principal forma de acesso a esses medicamentos por parte da população como a estudada. Neste contexto, as unidades de Saúde da Família poderão desempenhar papel relevante na instituição da cultura de uso de genéricos, tanto por parte dos prescritores quanto dos usuários dos serviços de saúde.

Conflito de interesses: não há.

Financiamento: não houve.

Colaboradores: M. Z. S. Vosgerau foi a responsável pela coleta, análise dos dados e redação do artigo; R. K. T. Souza participou na discussão dos resultados e revisão crítica do texto; D. A. Soares orientou a pesquisa e participou na discussão dos resultados.

Agradecimento: Em especial, a Darli Antonio Soares (in memoriam), pioneiro na área de saúde coletiva no Paraná, que contribuiu para que este trabalho e muitos outros pudessem ser desenvolvidos.

\section{Referências}

1. Ministério da Saúde. Avaliação da assistência farmacêutica: estrutura, processo e resultados. Brasília: OMS/OPAS/Ministério da Saúde; 2005.

2. Ministério da Saúde. Lei no 9.787, de 10 de fevereiro de 1999. 1999b. Disponível em http:/ / www.cff.org. br/Legisla\%C3\%A7\%C3\%A3o/Leis/lei_9787_99.html. [Acessado em 18 de dezembro de 2007]
3. Brasil. Agência Nacional da Vigilância Sanitária. Medicamentos: conceitos técnicos. Disponível em http:// www.anvisa.gov.br/medicamentos/conceito.htm. [Acessado em 22 de outubro de 2010]

4. Menda ME org. Manual médico: medicamentos genéricos. São Paulo: Lemos Editorial; 2002. 
5. Dias CRC, Romano-Lieber NS. Processo da implantação da política de medicamentos genéricos no Brasil. Cad Saude Publ 2006; 22: 1661-9.

6. Bertoldi AD, Barros AJD, Hallal PC. Generic drugs in Brazil: known by many, used by few. Cad Saude Publ 2005; $21: 1808-15$.

7. Rocha CE, Barros JAC, Silva MDP. Levantamento de dados sobre o conhecimento e informação acerca dos medicamentos genéricos em uma população de pacientes do serviço de saúde ambulatorial do Recife, Pernambuco, Brasil. Cad Saude Publ 2007, 23: 1141-50.

8. Faria MAS, Tavares Neto J. Conhecimento popular sobre medicamento genérico em um Distrito DocenteAssistencial do Município de Rio Branco, Estado do Acre, Brasil. Epidemiol Serv Saude 2006; 15: 37-45.

9. Brasil. Ministério da Saúde. Departamento de Atenção Básica. Saúde da Família terá reforço de US\$166,9milhões. Disponível em http://portal. saude.gov.br/portal/ aplicacoes/noticias/default. $\mathrm{cfm}$ ?pg=dspDetalheNoticia\&id_area $=124 \& C C_{-}$ NOTICIA=10524. [Acessado em 22 de outubro de 2010]

10. Associação Brasileira de Empresas e Pesquisa. Critério de classificação econômica Brasil 2000. Disponível em http://www.abep.org.br/codigosguias/ ABEP-CCBE.pdf. [Acessado em 19 de junho de2006]

11. Landry JA et al. Validation of two methods of data collection of self-reported medicine among the elderly. Gerontologist 1988; 28: 672-6.

12. Rozenfeld S, Valente J. Estudos de utilização de medicamentos - considerações técnicas sobre coleta e análise de dados. Epidemiol Serv Saude 2004; 13: 115-23.

13. World Health Organization. ATC/DDD Index 2005. 2005. Disponível em http://www.whocc.no/atcddd/. [Acessado em 20 de dezembro de 2006]

14. Ministério da Saúde. Departamento de Assistência Farmacêutica e Insumos Estratégicos. Relação Nacional de Medicamentos Essenciais 2006. 5 ed. Brasília: Brasil; 2007.

15. Secretaria Municipal de Saúde. Relação Municipal de Medicamentos essenciais 2002. Ponta Grossa; 2006.

16. Vieira FS, Zucchi P. Diferenças de preços entre medicamentos genéricos e de referência no Brasil. Rev Saude Publ 2006; 40: 444-6.
17. Monteiro WM, Melo GC, Massunari GK, Hübner DV, Tasca RS. Avaliação da disponibilidade de medicamentos genéricos em farmácias e drogarias de Maringá (PR) e comparação de seus preços com os de referência e similares. Rev Bras Cienc Farmac 2005; 41: 333-43.

18. Associação Brasileira das Indústrias de Medicamentos Genéricos. Mercado Mundial de Genéricos. Disponível em http://www.progenericos.org.br/ mercado.shtml. [Acessado em 10 de fevereiro de 2010]

19. Carvalho MCRD, Accioly Júnior H, Raffin FN. Representações sociais do medicamento genérico por consumidores residentes em Natal, Rio Grande do Norte, Brasil. Cad Saude Publ 2006; 22: 653-61.

20. Tobar F. Economía de los medicamentos genéricos en América Latina. Rev Panam Salud Publ 2008; 23: 59-67.

21. Rumel D, Nishioka AS, Santos AAM. Intercambialidade de medicamentos: abordagem clínica e o ponto de vista do consumidor. Rev Saude Publ 2006; 40: 921-7.

22. Lima-Costa MF, Matos DL, Camarano AA. Evolução das desigualdades sociais em saúde entre idosos e adultos brasileiros: um estudo baseado na Pesquisa Nacional Por Amostra de Domicílios (PNAD 1998, 2003). Ciência \& Saúde Coletiva 2006; 11(4): 941-50.

23. Carvalho MCRD, Accioly Júnior H, Raffin FN, Campos MN, Cruz MMC, Alves MKS. Representações sociais do medicamento genérico por farmacêuticos: determinação dos sistemas central e periférico. Cad Saude Publ 2005; 21: 226-34.

24. González CPV. Medicamentos genéricos y las políticas de medicamentos essenciales: uma alternativa para incrementar el acesso? In: Barros JAC org. Os fármacos na atualidade: antigos e novos desafios. Brasília: ANVISA; 2008.

25. Leyva R. Uso de medicamentos genéricos intercambiables en México. Salud Publ México 1999, 41: 435-6.

26. Luiza VL, Castro CGSO, Nunes JM. Aquisição de medicamentos no setor público: o binômio qualidade custo. Cad Saude Publ 1999; 15: 769-76.

27. Vosgerau MZS, Soares DA, Souza, RKT. Automedicação entre adultos na área de abrangência de uma Unidade Saúde da Família. Lat Am J Pharm 2008; 27(6): 831-8.

Recebido em: 26/03/10

Versão final reapresentada em: 28/10/10

Aprovado em: 16/11/10 